\title{
Implementación del modelo matemático para el sistema de control de una incubadora para aves utilizando la herramienta computacional MATLAB® - SIMULINK®
}

\author{
Yesenia Restrepo Chaustre ${ }^{1}$ y José Ricardo Bermúdez Santaella ${ }^{2}$
}

\section{Resumen}

En el presente artículo se describe la implementación del modelo matemático de una incubadora para aves, realizada por el ingeniero José Ricardo Bermúdez Santaella en su maestría "Automatización e instrumentación"[1], en donde se involucra el área de control y el manejo de la herramienta computacional MATLAB® - SIMULINK®.

De igual manera, se describe el proceso necesario para llevar a cabo la implementación del modelo matemático, teniendo como base las variables involucradas en la incubación de aves para realizar la selección de la estrategia de control, y adicionalmente el tiempo de respuesta, sensibilidad y robustez de la variable controlada.

Para finalizar se utiliza la herramienta computacional en la sintonización del controlador recurriendo al método de lazo abierto de Ziegler-Nichols[2] o curva de reacción.

Palabras Clave: MATLAB ${ }^{\circ}$ - SIMULINK®, temperatura, estrategia de control, PID.

\section{Abstract}

In the present article the implementation of the mathematical model of a incubator for birds is described, realised by the engineer Jose Ricardo Bermúdez Santaella in its masters "Automatization and instrumentation" [1], where it becomes jumbled the control area and the handling of computer tool MATLAB® - SIMULINK®.

Of equal way, the process necessary is described to carry out the implementation of the mathematical model, having as it bases the involved variables on the incubation of birds to realise the selection of the control strategy, and additionally the response time, sensitivity and robustness of the controlled variable.

To finish the computational tool is in use in the tuning of the controller resorting to the method of bow opened of Ziegler-Nichols [2] or curve of reaction.

Key Words: MATLAB® - SIMULINK®, temperature, control strategy, PID.

\footnotetext{
${ }^{1}$ Universidad Francisco de Paula Santander, yese_restrepo@hotmail.com

2Universidad Francisco de Paula Santander, jrbermud@hotmail.com
} 


\section{Introducción}

$\mathrm{M}$

$A T L A B \circledR$ es un lenguaje de muy alto nivel diseñado para cómputo técnico e integra en un mismo ambiente cálculos, visualización y programación. Algunos de los usos más comunes son: cálculos matemáticos, desarrollo de algoritmos, modelado, simulación, análisis de datos y desarrollo de interfaces gráficas. Junto a MATLAB® se ha desarrollado una colección de herramientas, algunas de ellas con enfoque a sistemas de control, procesado de señales, procesado de imágenes, lógica difusa, redes neuronales, simulación, optimización, finanzas y economía, entre otras. Estas herramientas se conocen como toolboxes y constituyen una parte importante de MATLAB ${ }^{\circledR}$ que permiten resolver una clase particular de problemas. [3].

La realización de la implementación de la estrategia de control del modelo matemático de una incubadora para aves fortalece los conocimientos adquiridos y sustentados en el área de control, por ello, se apoya en la utilización de la herramienta computacional MATLAB® - SIMULINK $®[3$, 4], utilizando el bloque del controlador PID [5,6] y el método de lazo abierto de Ziegler-Nichols [2] para la sintonización del mismo. Instrumento que servirá como soporte de las asignaturas del área de control, del programa académico Ingeniería Electrónica.

En efecto la implementación del modelo matemático, es un aporte ante la necesidad industrial y desarrollo de la región, mediante un proceso de modernización tecnológica en el sector avícola, y a su vez ubicar a la Universidad Francisco de Paula Santander como apoyo fundamental en la industria [7].

\section{Materiales y Métodos}

La herramienta computacional utilizada para el desarrollo de la implementación del modelo matemático del proceso de incubación en una incubadora para aves es el MATLAB® SIMULINK $®$, al obtener la implementación del modelo matemático utilizando esta herramienta, se procede a la selección de la estrategia tradicional más conveniente para este sistema, utilizando técnicas reconocidas como es el criterio de Nichols-Ziegler.

Simulink. El usuario puede crear sus modelos matemáticos a partir de una librería de componentes básicos, copiándolos de una ventana a otra, estableciendo las conexiones oportunas y dando valores a sus parámetros. La herramienta computacional SIMULINK ${ }^{\circledR}$ es utilizada para implementar el modelo matemático [7].

Sintonización con el método de lazo abierto [7]. El método utilizado para la sintonización del controlador es el método de lazo abierto o curva de reacción. Este método coloca el controlador en modo manual, los datos requeridos para el ajuste, se obtiene mediante la prueba de escalón que proporciona una curva de reacción como respuesta. Estos datos son los parámetros de $\mathrm{K}, \tau$, t0, obtenidos bien sea de un sistema de primer orden más tiempo muerto o de un sistema de segundo orden más tiempo muerto.

El método de sintonización a lazo abierto se realiza de esta forma:

- Se dispone el controlador en manual, y esperar que el proceso se estabilice.

- Realizar un cambio escalón en la señal de salida del controlador.

- Registrar la curva de respuesta del proceso.

Los procesos se expresan con una ecuación de transferencia de la forma:

$$
\frac{O(s)}{I(s)}=\frac{K e^{-t_{0} s}}{\tau+1}
$$

La otra ecuación es la de segundo orden, de la forma:

$$
\frac{O(s)}{I(s)}=\frac{K e^{-t_{o} s}}{\left(\tau_{1} s+1\right)\left(\tau_{2} s+1\right) \bullet \bullet \bullet\left(\tau_{n} s+1\right)}
$$

Los procesos de orden mayor son inicialmente aproximados a procesos de primer orden más tiempo muerto o procesos de segundo orden más tiempo muerto.

La figura 1, muestra la curva de reacción del proceso usando el método de los dos puntos, que aproxima un proceso de orden mayor a un proceso de primer orden. 
Figura 1. Curva de reacción del proceso usando el método de los dos puntos [7].

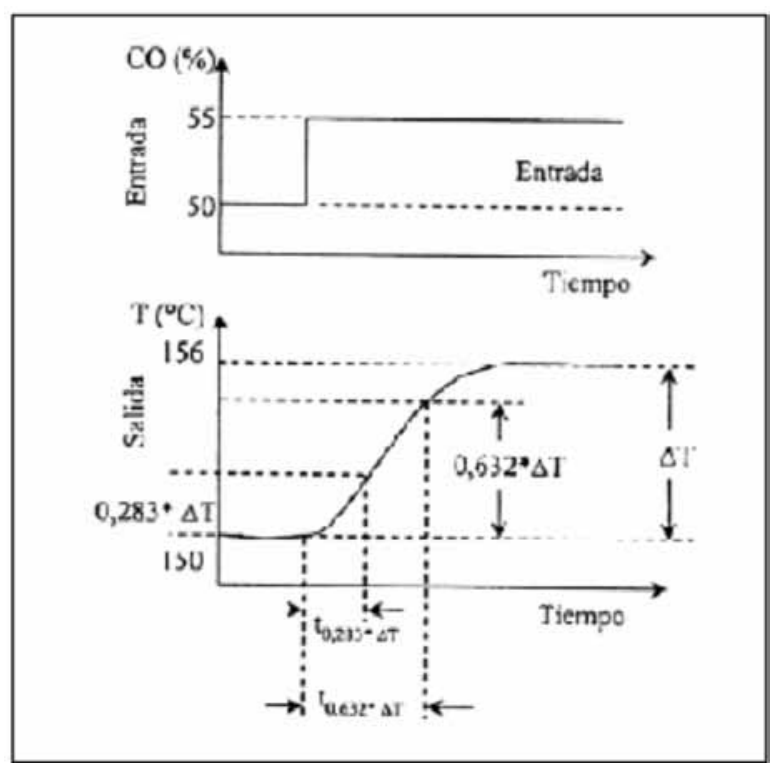

Uno de los métodos para obtener los valores de $\tau, t_{0}$, se denomina método de los dos puntos, y consiste en obtener dos puntos de los datos extraídos de la curva de reacción del proceso.

Estos dos puntos son:

- El tiempo que demora el proceso en alcanzar el 63.2\% del cambio total en la salida.

$$
(t /(0.632 \Delta O))
$$

- El tiempo que demora el proceso en alcanzar el $28,3 \%$ del cambio total en la salida.

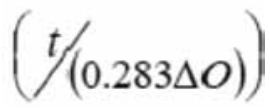

Al obtener estos dos puntos procedemos a reemplazar en las siguientes ecuaciones:

$$
\begin{aligned}
& \tau=1.5\left(\left.t\right|_{0.632 \Delta 0}-\left.t\right|_{0.283 \Delta 0}\right) \\
& t_{0}=\left.t\right|_{0.632 \Delta 0}-\tau
\end{aligned}
$$

El parámetro $\mathrm{K}$ (ganancia del proceso) debe estar en $\%, \tau$ (constante de tiempo) y $t_{o}$ (tiempo muerto) deben estar en minutos.

Método de Ziegler-Nichols a lazo abierto [7]. Al igual que el método de lazo cerrado, con los ajustes encontrados al aplicar este método, se intenta obtener una curva de respuesta de lazo cerrado que tenga una razón de amortiguador igual a 1/4. A partir de la tabla (Véase cuadro 1), se pueden determinar los coeficientes de ajuste a partir de los valores de $\mathrm{K}, \mathrm{t}_{\mathrm{o}} \mathrm{y} \tau$.

Nota: Cuando se utiliza Matlab, se debe hacer una conversión importante que es:

$$
\begin{gathered}
K_{p}=K_{c} \\
K_{i}=\frac{K_{c}}{T_{i}} \\
K_{d}=K_{c} T_{d}
\end{gathered}
$$

\begin{tabular}{|c|c|c|}
\hline Controlador & Parimetro de A uste & Ecuaciōn \\
\hline Proportional,? & $K_{p}$ & $\frac{1}{\mathrm{~K}}\left(\frac{\mathrm{t}}{\mathrm{t}}\right)$ \\
\hline Proporc:onal + Integnal, PI & $\begin{array}{l}K_{p} \\
{ }_{i}\end{array}$ & $\begin{array}{c}\frac{0,9}{K}\left(\frac{\mathrm{t}}{\tau}\right) \\
3,3 \mathrm{r}_{\mathrm{6}}\end{array}$ \\
\hline $\begin{array}{l}\text { Proportional + Integral } \\
\text { + Derivativo, PID }\end{array}$ & 4 & $\begin{array}{c}\frac{1,2}{\mathrm{~K}}\left(\frac{\mathrm{t}}{\tau}\right) \\
2,0,6\end{array}$ \\
\hline
\end{tabular}

Cuadro 1. Parámetros de sintonización de Ziegler-Nichols a lazo abierto [7].

\section{Implementación del modelo matemático de una incubadora para aves en SIMULINK® [7].}

Después del estudio, análisis y comprensión del modelo matemático propuesto por el Ing. José Ricardo Bermúdez Santaella [1,7], se da inicio a la implementación del mismo utilizando la herramienta computacional SIMULINK® .

En esta implementación se plasman las ecuaciones matemáticas [7, anexo B],en un bloque que se llama incubador, en el cual se involucra a la carga de huevos y las características físicas del medio como paredes, calentador, bandejas y flujo de aire; y un segundo bloque llamado huevo, en donde se envuelve la transferencia de energía presentada por la energía metabólica generada por el embrión, la convección, conducción, radiación y energía 
por evaporización, (ver figura 2), para mostrar el calor por conducción en el interior del huevo, calor generado por el huevo, temperatura de cáscara, temperatura del embrión y temperatura del incubador ( variable controlada).

Seguidamente, se ilustran los pasos para la implementación del modelo matemático de una incubadora para aves, propuesto por el Ingeniero José Ricardo Bermúdez Santaella[1], utilizando la herramienta computacional SIMULINK®.

El primer paso, consiste en plasmar las ecuaciones diferenciales que intervienen en el huevo y en el incubador [7, anexo A], utilizando la herramienta computacional de Simulink ${ }^{\circledR}$.

En el balance de energía del huevo, presentada en la figura 2, se involucra el balance de energía de la capa cáscara (bloque fucsia) y el balance de energía de la capa embrión (bloque rojo), en el cual se manipula la energía metabólica del embrión ( $\mathrm{EM}(\mathrm{t}))$, el calor por radiación del huevo ( $\operatorname{Qrad}(\mathrm{t})$ ) y el calor por evaporización del mismo ( Qevap(t)) para finalmente obtener el calor generado por el embrión en el proceso de incubación $(\mathrm{Qh}(\mathrm{t}))$.

Figura 2. Bloques que satisfacen el modelo matemático de una incubadora para aves[7].

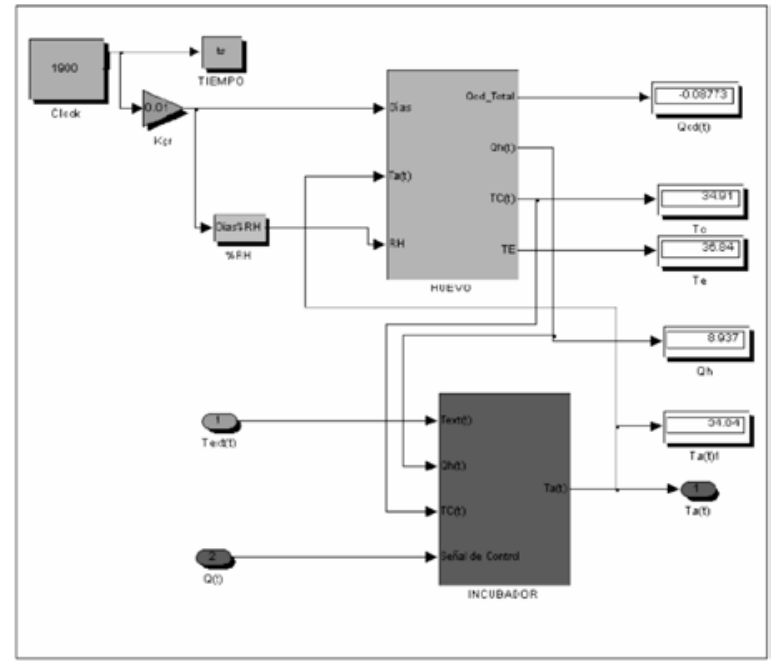

El segundo paso, consiste en realizar un archivo en ambiente editor de MATLAB® incluyendo las condiciones iniciales del sistema [7], descritos en la propuesta de su maestría por el ingeniero José Ricardo Bermúdez Santaella [1].
El tercer paso, consiste en la simulación del modelo matemático de una incubadora para aves, implementado en SIMULINK ${ }^{\circ}$, con el fin de obtener las curvas de la variable controlada ( temperatura del incubador - Ta ), en su estado estable, dependiendo de la especie a incubar [7, ver anexo $\mathrm{C}]$.

Al simular, el sistema mencionado en la figura 2, que involucra todas las ecuaciones y variables del modelo matemático[1], y teniendo en cuenta la temperatura de incubación para el pollo de $38^{\circ} \mathrm{C}$, temperatura de la variable controlada de $38^{\circ} \mathrm{C}$ ( Ta ), temperatura del ambiente exterior de $28^{\circ} \mathrm{C}$ ( Text ), variación de la humedad entre $100 \%$ y $55 \%$, en un intervalo de tiempo que abarca los 19 días de incubación con un sistema ON - OFF, se obtiene la siguiente figura.

Figura 3. Control de la temperatura del incubador [7].

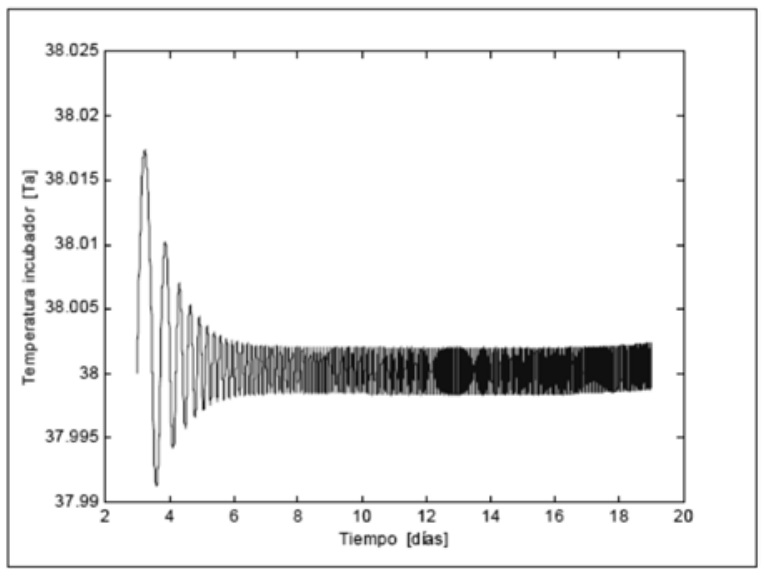

\section{Estrategia de control [7].}

Una vez analizado, estudiado e implementado el modelo matemático [1] utilizando la herramienta computacional MATLAB® - SIMULINK®, se procede a hallar la estrategia de control tradicional adecuada de este sistema. En donde, la variable fundamental para controlar el ambiente en el proceso de incubación es la temperatura del incubador ( Ta ).

Seguidamente, se utiliza el controlador tradicional P.I.D (Proporcional, Integral y Derivativo), que se encuentra en las librerías del SIMULINK®. En la figura 4 se observan los bloques que conforman el controlador. 
Figura 4. Modelo del control PID [7].

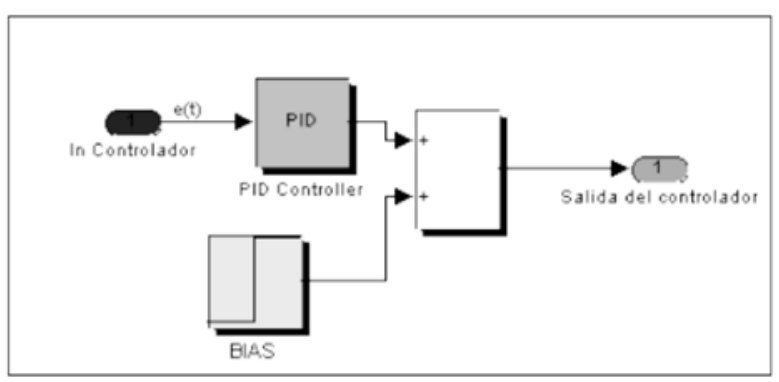

Para la selección y sintonización del controlador tradicional, se retoma el desarrollo de la implementación del modelo matemático [1,7], en un bloque llamado incubador, adicionando los cuatro bloques, llamados bloque del controlador, ejecutor, transmisor y convertidor, como lo ilustra la figura 5 .

Figura 5. Lazo cerrado [7].

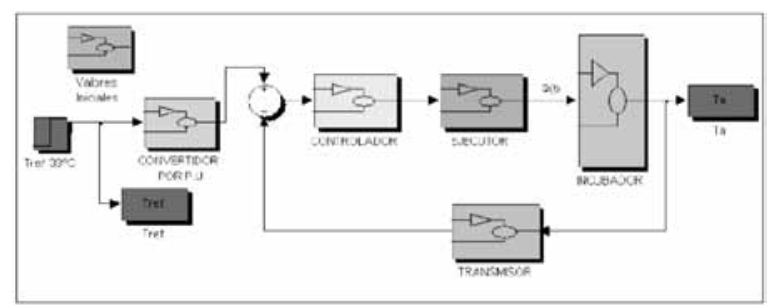

Para la sintonización del controlador tradicional adecuado para este sistema, se tomará como referencia la configuración presentada en la figura 6, con el fin de realizar el método de Ziegler - Nichols a lazo abierto, y obtener con esta configuración la curva de reacción para conocer las constantes que involucran la estrategia tradicional en sus respectivos modos de operación, como son:

- Modo Proporcional ( P ).

- Modo Proporcional-Integrativo ( PI ).

-Modo Proporcional-Integrativo-Derivativo PID ).

Figura 6. Sintonización del controlador.

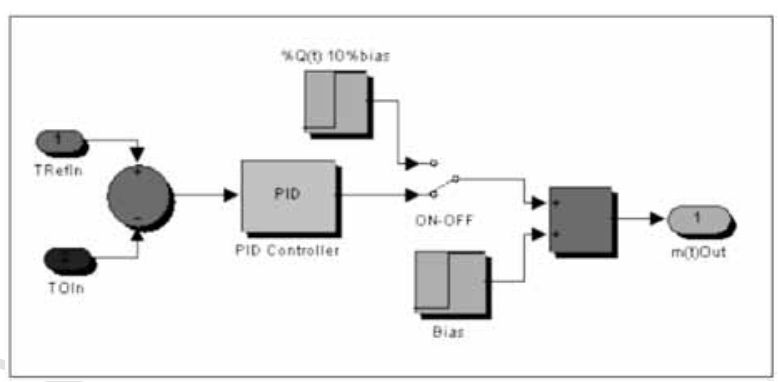

Para obtener la curva de reacción se realiza un escalón unitario al proceso en lazo abierto, es decir se elimina la salida del PID, dejándose solamente el bias, en el flujo de entrada con una variación del $10 \%$ ( 0.5 ) y se grafica la respuesta del sistema en la salida del transmisor.

\section{Resultados y Discusión}

Al realizar la sintonización de cada uno de los modos de controlador tradicional ( PID, P y $\mathrm{PI}$ ), se obtienen las constantes para los modos del controlador para lo cual se seleccionó un calentador de $200 \mathrm{~W}$ de máxima potencia para una carga de 200 huevos de pollo, de acuerdo a las muestras tomadas durante las simulaciones con diversas potencias nominales para el calentador ( $100 \mathrm{~W}, 150 \mathrm{~W}$ y $300 \mathrm{~W}$ ) y la variación de la temperatura externa del ambiente del incubador, Text, $28^{\circ} \mathrm{C}$, en la tabla 1 y tabla 2 , se dan a conocer los valores de $\tau$, tiempo muerto $\left(t_{o}\right)$ y los valores de las constantes [7].

Tabla 1. Valores proporcionados por la curva de reacción para una fuente de 200W [7].

\begin{tabular}{cc}
\hline $\mathbf{T}_{\text {ext }}$ & $\mathbf{2 8 ^ { \circ } \mathbf { C }}$ \\
\hline $\mathbf{t}_{\mathbf{1}}$ & 1.2014 \\
$\mathbf{t}_{\mathbf{2}}$ & 5.0294 \\
$\boldsymbol{\tau}$ & 5.742 \\
$\mathbf{t}_{\mathbf{0}}$ & -0.7126 \\
$\boldsymbol{K}$ & 6.632 \\
\hline
\end{tabular}

Una vez, conocidos los valores de las constantes del controlador, según la tabla 2, se introducen en el bloque PID generado por SIMULINK®, dando inicio a la simulación del sistema y teniendo como parámetro fundamental para la selección de la estrategia de control adecuada los diversos índices de comportamiento de la señal controlada, como son: tiempo de respuesta, sensibilidad, estabilidad y robustez de la variable controlada.

La figura 7 presenta el comportamiento de la temperatura del incubador, cuyo valor debe mantenerse para un óptimo funcionamiento del incubador en $38^{\circ} \mathrm{C}$, para el pollo, y para comparar cual es la estrategia de control más adecuada, según los índices de comportamiento mencionados anteriormente, se introdujo una señal de disturbio de $+/-25 \%$ sobre la temperatura externa. 
Tabla 2. Constantes del controlador tradicional [7].

\begin{tabular}{|c|c|}
\hline $\begin{array}{l}\text { CONSTANTES DE LOS MODOS DEL } \\
\text { CONTROLADOR TRADICIONAL }\end{array}$ & $\begin{array}{l}\mathrm{T}_{\mathrm{EXT}}= \\
28^{\circ} \mathrm{C}\end{array}$ \\
\hline Proporcional & -1.215 \\
\hline $\begin{array}{l}\text { Proporcional } \\
\text { Integrativo }\end{array}$ & $\begin{array}{r}-1.0935 \\
4.6512\end{array}$ \\
\hline $\begin{array}{c}\text { Proporcional } \\
\text { Integrativo } \\
\text { Derivativo }\end{array}$ & $\begin{array}{l}-1.458 \\
1.023 \\
0.5195\end{array}$ \\
\hline
\end{tabular}

Figura 7. Temperatura en el incubador ( Ta ) para una temperatura de ambiente externo de $28^{\circ} \mathrm{C}$ [7].

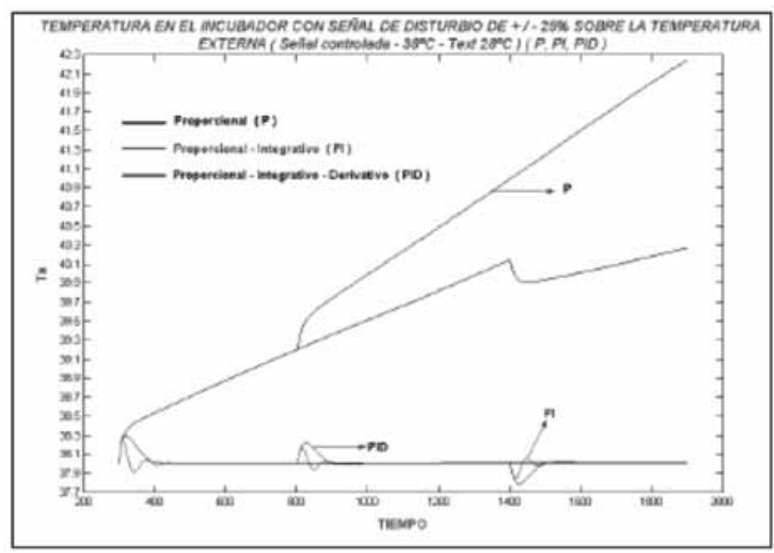

De acuerdo al análisis de los índices de comportamiento de la variable controlada $\mathrm{Ta}$ ilustrada en la figura 7, la estrategia de control más conveniente para implementarlo en el incubador es el controlador tradicional el modo PI ( Proporcional - Integrativo ), en el cual se observa que los picos de disturbio son menores al controlador P y PID.

\section{Conclusiones}

El proceso para la sintonización del controlador tradicional utilizado en el desarrollo de este proyecto fue el Método de Ziegle - Nichols a lazo abierto utilizando la curva de reacción.

La estrategia de control tradicional seleccionada fue la PI, de acuerdo a los índices de comportamiento como: tiempo de respuesta, sensibilidad y robustez, para con ello obtener un óptimo funcionamiento de un incubador para aves.

Conlaimplementación de este modelomatemático, en SIMULINK, se seleccionan los componentes físicos que conforman una incubadora para aves (características y dimensiones de las paredes, fuente de energía, características del ventilador, bandejas de huevos, sensores, etc. ), además, de desplegar una presentación amigable es también versátil y flexible a la variación de algunos términos durante la simulación.

Una de las herramientas más idóneas, sencillas, versátil, flexible y con una presentación amigable para la implementación de ecuaciones integrodiferenciales, es la herramienta computacional SIMULINK ${ }^{\circledR}$. La herramienta computacional MATLAB $\AA$ - SIMULINK $\AA$ presenta un ambiente de programación gráfica, que permite el desarrollo de aplicaciones que se ejecutan con una rapidez similar a las de programas realizados en $\mathrm{C} / \mathrm{C}++$, ya que su compilador es de 32 bits, lo que lo convierte en un software altamente versátile y de gran utilidad durante el desarrollo del proyecto.

\section{Bibliografía}

[1] BERMÚDEZ S. José R. Propuesta de un modelo matemático de una incubadora para aves. Mérida: ULA. 2000. 387 p.

[2] ALFARO RUIZ Víctor M., Actualización del método de sintonización de controladores de Ziegler y Nichols. En revista: Ingeniería 15 (1,2): 39-52, ISSN: 1409-2441, 2005, San José, Costa Rica. http://eie.ucr.ac.cr/uploads/file/documentos/ pub_inv/ articulos/valfaro05A.pdf

[3] BÁEZ LÓPEZ David, MATLAB con aplicaciones a la ingeniería, física y finanzas, 2006, Alfaomega, 445p.

[4] RAJAGOPALAN Arun, WASHINGTON Gregory, SIMULINK TUTORIAL, 2002, http:// sts.bwk.tue.nl/7y700/readers/.\%5CA06_Tutorial_ SimuLink_ENG.pdf

[5] SMITH, CORRIPIO, Control automático de procesos, 1995, Limusa Noriega Editores, 717p.

[6] DORSEY John, Sistemas de control continuos y discretos, 2005, Mc Graw Hill, 630p.

[7] RESTREPO CH. Yesenia, Implementación del modelo matemático para el sistema de control y supervisión de una incubadora para aves, TIE 629.8312 R436i, 2005, 148p, Tesis para optar al título de Ingeniero Electrónico. 